\title{
Autoimmunity Highlights: a bridge between the clinic and the laboratory
}

\author{
Nicola Bizzaro
}

Autoimmune diseases include a wide range of diseases which differ in terms of demographic profile and clinical manifestations. At the same time, despite the diversity in their presentation and progression, autoimmune diseases share common pathophysiological mechanisms which allow their characterization as a specific subdiscipline within the field of internal medicine.

Besides the numerous rheumatic diseases, an autoimmune pathogenesis is recognized in many diseases of the endocrine, dermatological, neurological, haematological, hepatic and gastrointestinal systems and organs, so that no medical specialty is excluded from the field of autoimmunity. Although many of these diseases taken individually are very rare, taken together they involve up to $10 \%$ of the population, with prevalence and incidence on the rise. Their social and economic impact is, therefore, substantial.

\footnotetext{
N. Bizzaro

Editor-in-Chief

Laboratorio di Patologia Clinica

Ospedale Civile

Tolmezzo (UD)

E-mail: nicola.bizzaro@ass3.sanita.fvg.it
}

For these reasons, both basic research and clinical research on autoimmune diseases has become ever more important for reducing the impact of these conditions. From epidemiological and physiopathological studies we can expect significant progress in understanding the mechanisms which lead to the onset of autoimmune diseases as well as the mechanisms involved in disease progression. From clinical studies we can expect clearer information on the significance and advantages of early diagnosis even in asymptomatic subjects. The development of new and more effective therapeutic strategies is also the subject of continuous research. Even in the fields of imaging and laboratory diagnostics, many notable achievements have been made and more are expected.

Recent advances in in vivo imaging technologies such as positron emission tomography (PET) and magnetic resonance imaging (MRI), using contrast agents which label specific cells, have the potential for mapping these cells in living tissues and monitoring their functions. In the years to come, the application of these new high-resolution imaging strategies will eventually provide a powerful means for noninvasive detection, diagnosis and monitoring of immunological diseases.

Laboratory diagnostics of autoimmune diseases is another area of great relevance. Even in this specific sector, over the last 20 years progress has been remarkable. Immunometric tests with recombinant or synthetic antigens have come alongside the traditional immunofluorescence techniques and have progressively replaced the first-generation methods (precipitation, etc.) which are cumbersome and not very practical. Thus the sensitivity and specificity of results has improved greatly. The future will offer new technologies, such as genomics and proteomics, and the use of sophisticated methods based on microchip technology and mass spectrometry. These 
diagnostic systems characterized by the capacity to furnish multiparameter results with a throughput rate that was unimaginable only a few years ago, and the identification of putative biomarkers and signature protein expression patterns characteristic of specific autoimmune diseases, will probably change diagnostic and therapeutic strategies fundamentally.

Autoimmunity Highlights is born with a clear purpose: to witness these changes and make them available to the medical community, giving special attention to the links between clinical practice, diagnostics and laboratory research. Considering the great number of diseases with an autoimmune pathogenesis, the field of interest of Autoimmunity Highlights is necessarily very wide. Given these factors, it has been decided to appoint to the journal a large number of internationally recognized scientists who have expertise in the field of autoimmune pathology. They will undertake the function of Section Editors. The composition and the multidisciplinary nature of the Board is a guarantee of the quality of the journal and a basis for the success of Autoimmunity Highlights.

I am confident that this journal, made possible by the interest and competence of the publisher, Springer, will become known as one of the best scientific journals in the field of autoimmune disease and will find a close following among the many researchers, scientists and clinical physicians concerned with autoimmune diseases.

Papers will be selected on the basis of current importance to the field, novelty, and relevance to the potential readership. Our principal effort will be to ensure both authors and readers of the integrity of the editorial process, and that the journal follows the highest ethical standards. According to this policy, disclosure statements for the Editor in Chief and all Editorial Board members will be available for the scientific community on the journal website.

On behalf of the Editorial Board, of Springer, and myself personally, I offer our complete commitment to the achievement of these objectives. 